G276(P) IMPACT OF A REGIONAL PAEDIATRIC TUBERCULOSIS NETWORK

A Sinha, S Dixon, L Turnbull, F Child. Department of Paediatric Respiratory Medicine, Royal Manchester Children's Hospital, Manchester, UK

10.1136/archdischild-2020-rcpch.239

Introduction Tuberculosis (TB) incidence in England and Wales has fallen by $44 \%$ since 2011 with England now classed as a low incidence area. This means that individual clinicians are seeing fewer cases of $\mathrm{TB}$ and experience is falling. Working with public health and the TB Control Board we have created a regional paediatric $\mathrm{TB}$ network consisting of tertiary and secondary TB teams across an area with varying TB incidence. The aim of this network was to improve and standardise care, reduce diagnostic delay and increase our shared experience.

Method The network has been commissioned for and encourages engagement and referrals from DGH paediatricians and TB nurses. It includes a weekly virtual TB clinic and enhanced Paediatric annual cohort audit with feedback of learning points at regular stakeholder education days.

Results The network and virtual clinic have been running for 5 and 2 years respectively. Seventy patients have been discussed at the virtual clinic: in $57 \%$ of children this led to a change to investigation, diagnosis or management. Four children had a change of diagnosis from latent TB infection (LTBI) to active disease. The clinic has been able to cover gaps in service supporting solo clinicians and TB nurses and enabling $53 \%$ of children to have their care closer to home. Service delivery issues such as adult physicians looking after children without paediatric input and failure to provide adequate chemoprophylaxis in high risk children have been identified and addressed. Reasons for diagnostic delay have been identified to be different in different areas allowing site appropriate solutions to be explored. Focussed education sessions on safeguarding, pharmacy and drug delivery have been provided. Communication has improved region-wide and experience is increasing.

Conclusion The TB network has been well received by TB teams across the region. It is a great example of collaborative working, enabling resolution of service issues and improved quality of care for our patients.

\section{G277(P) CHILD PROTECTION EXPERIENCE AND TRAINING OF PAEDIATRIC JUNIOR DOCTORS - A REGIONAL STUDY}

${ }^{1} S$ Hussain, ${ }^{2} S$ Hosdurga. 'Community Child Health, University Hospitals Bristol, Bristol, UK; ${ }^{2}$ Community Child Health, Sirona Community Care and Health, Bristol, UK

\subsection{6/archdischild-2020-rcpch.240}

Background Paediatricians carry out medical assessments to determine and document harm following suspicion of child maltreatment and contribute for multiagency assessments. This involves making emotionally challenging decisions. Difficulties faced by paediatricians working in child protection (CP) have known to cause psychological morbidity and reservations to proactively engage with safeguarding process.

Aims

- To assess the CP experience and training of paediatric junior doctors at South Western and Peninsula Deanery

- To identify if this experience affects their emotional/mental health.
- To identify if safeguarding proforma assists trainees to have a structured approach to CP medical examination

Methods The survey questionnaires were distributed to 31 level 2 and 3 Paediatric trainees during deanery teaching days in February 2019. Data collected included: demographics, Level of safeguarding training, experience of dealing with CP cases, the supervision and support and a free text for any recommendations. A new safeguarding proforma was designed by collaborating ideas from various regional centres and $\mathrm{RCPCH}$ companion to see if this assists trainees for a structured medical assessment and report writing.

Results Thirty one responses were included. Majority had level 3 safeguarding training (29/31) and were aware of safeguarding guidance (25/31). 20\% of the trainee's felt that their CP experience did not fulfil their learning needs (excluding number of responders marked this question not applicable). 39\% described safeguarding experience being negative.35\% reported anxiety before the child protection on call and 29\% felt low afterwards. Majority commented that the safeguarding proforma will be a useful supportive tool. Thought provoking comments, modified CP proforma, full results, and recommendations will be shared during the presentation.

Conclusions The CP work needs good diagnostic, communication and team working skills. Good experience, training, supervision and additional supportive tools are key to get everything right and for psychological wellbeing of the junior trainees. Trainees should get opportunities to learn from their clinical experience in supportive environment to be competent and confident. As CP is a national and a global priority, some of the recommendations are applicable widely across the regions, trusts and organisations.

\section{G278(P) DEVELOPMENT OF A GUIDELINE FOR MEDICAL CARE OF CHILDREN WITH DOWN SYNDROME, FROM A PAEDIATRIC INTENSIVE CARE UNIT PERSPECTIVE}

R Poole, C De Munter. Paediatric Intensive Care Unit, St Mary's Hospital, London, UK

\subsection{6/archdischild-2020-rcpch.241}

Aims Down syndrome (DS) is the most common genetic cause of cognitive disability, with a worldwide incidence between 1 in 1000 and 1 in 1100 live births. Children with DS have characteristic physical features, with an increased incidence of congenital or acquired medical conditions. The Down Syndrome Medical Interest Group (DSMIG) have produced guidance for essential basic medical surveillance in children with DS. Children with DS have also been shown to respond differently to critical illness, requiring a higher proportion of organ support than expected by initial disease severity. This project aimed to produce a hospital guideline for medical care of children with DS, whilst also taking into account specific considerations for care of a child with DS in PICU, to standardise and improve the quality of care.

Methods Involvement in medical care of children with DS admitted to PICU revealed that despite the availability of clear DSMIG guidance, there was no hospital guideline for children with DS. Furthermore, in terms of PICU care, key considerations were anecdotally noted, yet these differed between consultants and there was no PICU-specific guideline to outline a gold standard of care. DSMIG guidance was reviewed and PICU consultants approached for key specific considerations 
for care of a child with DS on PICU. A guideline was produced, edited and reviewed at a hospital guideline meeting, and agreed on by all PICU consultants.

Results Using DSMIG guidance, a hospital guideline could be produced for medical care of children with Down Syndrome, with additional specific requirements for PICU. Key PICU-specific considerations were noted and agreed on by a consultant team in terms of: airway; respiratory; cardiovascular; neurological; and infection.

Conclusion An admission to PICU can be a window of opportunity to ensure a child with DS is receiving the correct support from a multidisciplinary team, whilst there are also key PICU-specific considerations for their care. An SHO working under different leaders has an opportunity to develop a guideline to define and standardise a gold standard of care.

\section{G279(P) THE DIAGNOSIS OF ASTHMA IN CHILDREN IN PRIMARY CARE AND THE EMERGENCY DEPARTMENT}

A Oswal. Department of Paediatrics, Southend University Hospital, Southend, UK

\subsection{6/archdischild-2020-rcpch.242}

Aims This audit was undertaken to measure compliance of the diagnosis of asthma in children to NICE guidelines (Age over 5 and documented objective evidence of asthma). In the primary care audit, the aims were to review the age at diagnosis and the percentage of children in whom objective evidence of asthma was documented. In the emergency department (ED) audit, the aims were to review at the age at diagnosis, whether any previous evidence of asthma was documented, and whether the child was discharged from ED.

Methods A snapshot audit at a general practice surgery, was undertaken of children (less than 16 years old) with a coded diagnosis of 'Asthma', to review the age at diagnosis and whether any objective evidence of asthma was documented at diagnosis.

50 consecutively cases of asthma in children presenting to the emergency department (ED) in a district general hospital were then retrospectively audited to review the age at presentation, whether there was evidence of previous asthma diagnosis and whether the patient was discharged from ED.

Results In the GP surgery, there were 83 coded cases of asthma in children. 51 children (61\%) were diagnosed under 5 , with 9 children (11\%) who had objective evidence of asthma documented at diagnosis.

In the ED, 50 cases were collected of which 4 were excluded as they were referred to the other services, or for incorrect coding for unrelated diagnoses. Of the 46 remaining, 14 were under the age of $5(30 \%)$ and 27 diagnoses conformed to NICE guidelines (59\%). 33 children (72\%) were discharged from ED to their GP, 20 of whom had correct diagnoses (61\%).

The coding system in ED did not allow for separate coding of viral induced wheeze and asthma hence the 19 incorrect diagnoses likely represented viral wheeze.

Conclusions The most likely reason for the apparent low rate of correct diagnosis is the significant challenge of obtaining objective evidence of asthma in children. This audit also adds evidence to suggest that discharging children from ED with incorrectly coded diagnoses can further confound the already complex issue of diagnosing asthma in children.

\section{G280(P) BREATHING PATTERN DISORDERS: CHARACTERISTICS AND OUTCOMES OF CHILDREN ATTENDING A SECONDARY CARE RESPIRATORY CLINIC}

${ }^{1}$ TP Newson, ${ }^{2}$ A Elias. ${ }^{1}$ Paediatric Department Kent and Canterbury Hospital East Kent Hospitals NHS University FT, Canterbury, UK; ${ }^{2}$ Speech and Language Therapy Department, Canterbury and Kent Community Trust, Canterbury, UK

\subsection{6/archdischild-2020-rcpch.243}

Aim Breathing Pattern Disorders (BPD) previously termed dysfunctional breathing are due to functional abnormalities of the thoracic or laryngeal mechanics of respiration that result in inefficient breathing and a variety of symptomatology. Symptoms such as dyspnoea are significantly handicapping and disproportionate to extent and severity of any associated disease e.g. asthma. Paediatric studies of BPD and outcomes are few yet we are increasingly recognising children attending our respiratory clinic and referring patients for specialist speech and language therapy (SALT). We therefore reviewed our experience.

Methods Retrospectively we analysed health records of 18 children with BPD referred to specialist SALT between 2015 to 2018 from a respiratory clinic.

Results Age range 11-16 years (median 14 with 14 female, 4 male). Co-morbidities 16 Asthma,2 Oesophageal atresia \& TOF repair, 1Chronic Regional Pain Syndrome, 1Chronic Fatigue Syndrome. Commonest BPD was induced laryngeal obstruction (ILO) in 16 patients (exercise induced EILO in 15). Other types of BPD included hyperventilation in 7; psychogenic cough 8 and dysphonia1. A single BPD was present in 6 (EILO) with two or more in 10 patients. Inducers of BPD were exercise 16; bullying 3 ; anxiety 14 ; stress 11 ; weather 3; posture 1; odours 3. Majority had significant school absences (1-24 months). Delay in diagnosis (1-24 months median 6 months). Emergency calls in 14 with one patient with ILO needing ventilation. Follow up minimum at 1 year 2 complete resolution, 14 good control of BPD and more confident with improved morbidity and school attendance; 2 no improvement; 1 lost to follow up. De- escalation of asthma treatment in 16 patients.

Conclusion BPD are important to recognise and treat early to limit the significant morbidity that adversely impacts quality life. The establishment of a referral pathway with early input of a specialist SALT allows rapid resolution of symptoms in many and prevents exhaustive unnecessary investigation and those with co-existing asthma inappropriate escalation of treatment.

\section{G281(P) IMPROVING PARENTAL UNDERSTANDING OF CHRONICNEONATAL LUNG DISEASE (CNLD) AND SATISFACTION WITH INFORMATION PROVISION REGARDING CNLD AND HOME OXYGEN PATHWAY}

${ }^{1} \mathrm{~L}$ Jones, ${ }^{1} \mathrm{R}$ McCrum, ${ }^{1} \mathrm{~S}$ Smith, ${ }^{1} \mathrm{M}$ Bharadwaj, ${ }^{2} \mathrm{~S}$ Mulla. 'Medical education, Kings College London, London, UK; ${ }^{2}$ Neonatology, East Kent University Hospitals NHS Trust, Ashford, UK

\subsection{6/archdischild-2020-rcpch.244}

Background CNLD is a multifactorial condition affecting premature infants and remains a major source of morbidity and mortality for extremely low birth weight infants. The management of the disease is a large burden to the child's family due to its long and complicated multidisciplinary care-pathway. Therefore, it is vital to fully educate parents 